\title{
Current Trends in Foodborne Human Listeriosis
}

\author{
Atin Datta, Laurel Burall \\ Center for Food Safety and Applied Nutrition, US Food and Drug Administration, Laurel, MD 20708, USA
}

\begin{abstract}
Human listeriosis results from the ingestion of foods contaminated with Listeria monocytogenes (Lm). About 1600 listeriosis cases are reported every year in the USA with $>95 \%$ hospitalization and $15-20 \%$ death. The proportions of persons with listeriosis hospitalized and who die are very similar in Europe with slightly higher rates in Scandinavian countries. The occurrence of disease requires adaptation, survival, and usually growth of $L m$ in foods before consumption by members of the susceptible population. Despite concerted efforts by the food safety community, the disease incidence has not changed significantly since 2001 and remains higher than the Healthy People 2020 target of 0.2 cases per 100,000 individuals.

In recent years, human listeriosis cases have been reported to involve non-typical foods, e.g. celery, cantaloupe, caramel apple, frozen vegetables and ice cream. In some outbreaks, a few infected individuals were considered outside the realm of the standard vulnerable population group. Our recent work with the outbreak associated with ice cream samples, indicated that a low-level contamination in a food that does not support growth can cause listeriosis in highly susceptible populations. Separately, using a combination of polymerase chain reaction (PCR)-based serotyping and whole genome sequencing (WGS)-based analyses; we have discovered that a genetic variant of the serotype $4 \mathrm{~b}$ strain, called $4 \mathrm{bV}$, was responsible for 3-4 recent outbreaks in the USA. Three of the four products associated with these outbreaks were grown in a small geographical region of the USA while the fourth was never linked to a specific grower, but rather a processing facility. These $4 \mathrm{bV}$ strains contain a $6.3 \mathrm{~kb}$ DNA fragment normally associated with lineage II $L m$ strains. The significance of this DNA fragment in the serotype $4 \mathrm{~b}$ background is currently being investigated. This article reviews current listeriosis outbreaks with an emphasis on the expansions in food niche, case demography and genotypes of $\mathrm{Lm}$. The discussion raises important questions about $\mathrm{Lm}$ adaptation in different foods and environments and the role of certain genotypes in such adaptation and disease outcome.
\end{abstract}

Key words: human listeriosis, new food types, Listeria monocytogenes, new serotypes/genotypes

\section{Introduction}

Human listeriosis, caused by Listeria monocytogenes $(L m)$, is a major foodborne disease which causes severe invasive infections (meningitis and sepsis) and, in some instances, gastroenteritis. The febrile gastroenteritis is a self-limiting disease which has been reported to occur in healthy persons and presents with fever, watery diarrhea, nausea, headache, and pains in joints and muscles. Several reported outbreaks of febrile gastroenteritis due to $\mathrm{Lm}$ have been reported in different continents ${ }^{1}$. The invasive form of the disease, on the other hand, most often affects immunocompromised persons, including the elderly, neonates, and persons with underlying diseases associated with reduced immune function. During pregnancy, listeriosis most often presents in the mother as a mild febrile illness. In the USA, approximately 1600 cases of listeriosis are reported annually resulting in 250 deaths ${ }^{2}$. The high fatality rate (about $20 \%$ )

Received: 1 November 2017; Accepted: 28 February 2018; Published online: 30 March 2018

Corresponding author: Atin R. Datta, Ph.D. CFSAN, US Food and Drug Administration, Laurel, MD 20708, USA (atin.datta@fda.hhs.gov)

The contents of this article reflect solely the view of the author(s).

This paper was presented at the 12th International Symposium "Toxins, Pathogens, and Foods: Challenges and Opportunities for Public Health" organized by the Toxic Microorganisms Panel of the United States-Japan Cooperative Program on Development \& Utilization of Natural Resources.

Abbreviations: BLAST: Basic Local Alignment Search Tool, CFSAN: Center for Food Safety and Nutrition, Lm: Listeria monocytogenes, MPN: most probable number, MVLST: Multi Virulence Loci Sequence Typing, PCR: polymerase chain reaction, PFGE: Pulsed Field Gel Electrophoresis, WGS: Whole Genome Sequencing 
and the hospitalization rate ( $>95 \%)$ make listeriosis one of the deadliest foodborne diseases. According to the Centers for Disease Control and Prevention, reported rates of human listeriosis in the USA have remained steady over the last few years, and if no changes occurs in the next few years, the rate will exceed the Healthy People 2020 target of 0.2 cases per 100,000 populations ${ }^{3)}$, suggesting the need for additional information and approaches to prevent this infection.

$\mathrm{Lm}$ is a Gram-positive, micro-aerophilic, non-spore forming organism ubiquitously present in the environment and has been detected in most raw agricultural products and many dairy products, including cheeses and ice cream. The organism is known to be resistant to high salt and grows well at refrigerated temperatures provided other conditions are suitable ${ }^{4}$. The resistance to high salt and the ability to grow at refrigerated temperatures makes this organism hard to control by standard preservation and storage conditions. It is often assumed that because of these properties Lm has an advantage over other organisms, which are more inhibited than $\mathrm{Lm}$ in high salt condition and in refrigerated temperatures as $\mathrm{Lm}$ is more resistant to these conditions. The genus Listeria is currently comprised of 17 species $^{5)}$ of which $L m$ and $L$. ivanovii are the only pathogens, although human infection by L. ivanovii is rarely reported ${ }^{5)}$. Based on somatic and flagellar antigens, $L m$ can be grouped into 13 serotypes although the clear majority of infections are caused by serotypes $1 / 2 a, 1 / 2 b$ and $4 b^{6,7)}$. Because of the complexity involved in classical serotyping by antigen-antibody reaction, several DNA based methods have been developed which depend on the presence/absence of genetic sequences. Using a PCR-based method, Doumith et al. ${ }^{8)}$ first reported a sero-grouping approach which resulted in formation of five serogroups- $1 / 2 \mathrm{a}-3 \mathrm{a}, 1 / 2 \mathrm{~b}-3 \mathrm{~b}-7,1 / 2 \mathrm{C}-3 \mathrm{c}, 4 \mathrm{~b}-4 \mathrm{~d}-4 \mathrm{e}$ and nontypeable, which don't fall into any of the other groups. Burall et al. ${ }^{9)}$ combined this method and a simple slide agglutination method to further divide the first three groups into $1 / 2 \mathrm{a}, 1 / 2 \mathrm{~b}$, $1 / 2 c, 3 a, 3 b-7$ and 3c. This PCR-based method also resulted in uncovering a subgroup of $4 \mathrm{~b}$, called $4 \mathrm{~b}$ variant, $4 \mathrm{bV}^{10)}$ or IVb-v1 ${ }^{11)}$. The $4 \mathrm{bV}$ group contains a $6.3 \mathrm{~kb}$ DNA fragment, not found in typical $4 \mathrm{~b}$ strains but instead is found to be associated with $1 / 2 a-3 a$ and $1 / 2 c-3 c$ strains of $L m$. Although the gene composition and organization of this $6.3 \mathrm{~kb}$ DNA and putative annotated functions have been elucidated, a limited effort to unravel its biological significance has been unsuccessful ${ }^{10)}$. Although all $\mathrm{Lm}$ strains are considered pathogenic, it is not clear why certain serotypes are over-represented among clinical isolates and why certain serotypes are over represented among food and environmental isolates. Assessment of the virulence potential of $\mathrm{Lm}$ strains using several in vitro and in vivo models has not been very successful in discerning any strain-to-strain or serotype-to-serotype differences in virulence potential although there are distinct genetic footprints associates with the different serotypes ${ }^{12)}$. The lack of a sensitive model for virulence assessment has also hampered our attempt to obtain a realistic picture of the infectious dose and the effect of food matrices on Listeria virulence ${ }^{13)}$.

$L m$ contains a single chromosome of about $3 \mathrm{MB}$ with the occasional presence of some plasmids and/or a few phage sequences. The relatively small genome length, $3 \mathrm{MB}$ vs 4.5 MB for E. coli and Salmonella, may be related to a lack of diversity (synteny) of the genome, although a recent publication by Chen et al. ${ }^{14)}$ questions that notion. The major virulence genes of $L m$ and L. ivanovii are in a $10 \mathrm{~kb}$ pathogenicity island (LPI) while few others are scattered around the chromosome. Two global transcription regulators, $p r f A$ for virulence genes and $\operatorname{sig} B$ for stress related functions, have been well characterized as have several of their regulatory targets. However, the significance of virulence genes in overall disease outcome and their impact on infectious dose along with the significance of $\operatorname{sig} B$-controlled genes in environmental adaptation are little understood. From a food contamination and preservation standpoint, the genetic controls underlying salt and acid tolerance, growth at refrigerated temperature, ability to form biofilm and how $L m$ escapes standard sanitary processes, remains very poorly understood.

A close look at the major invasive listeriosis outbreaks (Table 1) shows some important trends associated with food and serotype distribution starting with the coleslaw outbreak in Canada in 1980-1981. Starting from early 1980s and the next 20 years or so, dairy and meat products constituted the major food groups and serotype $4 \mathrm{~b}$ was the major serotype. This pattern, however, changed considerably as we have witnessed many more outbreaks involving strains of serogroup $1^{15)}$. Additionally, in the last few years we have witnessed many more outbreaks involving fresh produce and the potentially increased involvement of serotype $4 \mathrm{bV}$ strains. Understanding these trends would be useful in formulating policy guidelines which might reduce future outbreaks. A brief discussion of a few of these recent outbreaks highlights how policy guidelines could be affected by this increased understanding.

\section{Listeriosis Outbreak Related to Ice Cream}

In the early 2015, a listeriosis outbreak USA was reported involving 5 hospitalized patients in Kansas City. All the patients were reported to have drunk milkshakes made with individual pre-packaged ice cream servings. Three of the five patients died with listeriosis, as well as severe 
Table 1. Major invasive listeriosis outbreaks*.

\begin{tabular}{|c|c|c|c|c|}
\hline Year & Location & Cases (\% Mortality) & Food & Serotype \\
\hline $1980-81$ & Canada & $41(34)$ & Coleslaw & $4 b$ \\
\hline 1983 & Massachusetts & $49(29)$ & Pasteurized Milk & $4 b$ \\
\hline 1984 & Switzerland & $57(32)$ & Soft Cheese & $4 b$ \\
\hline 1985 & California & $142(34)$ & Jalisco Cheese & $4 b$ \\
\hline $1987-89$ & UK & 823 & Pate & $4 b$ \\
\hline 1992 & France & $279(30)$ & Rillettes (Pork) & $4 b$ \\
\hline 1998-99 & USA & $101(21)$ & Hot Dog, Deli Meats & $4 b$ \\
\hline 1998-99 & Finland & $25(24)$ & Butter & $3 a$ \\
\hline 2000 & USA & $29(7)$ & Deli Turkey Meat & $1 / 2 \mathrm{a}$ \\
\hline 2008 & Canada & $57(40)$ & Deli Meat & $1 / 2 \mathrm{a}$ \\
\hline 2011 & USA & $147(33)$ & Cantaloupe & $1 / 2 \mathrm{a}, 1 / 2 \mathrm{~b}$ \\
\hline 2012 & USA & $22(25)$ & Ricotta Salata Cheese & $1 / 2 \mathrm{a}$ \\
\hline 2013 & USA & $6(33)$ & Soft Cheese & $4 b$ \\
\hline 2014 & USA & $8(12)$ & Soft Cheese & $1 / 2 b$ \\
\hline 2014 & USA & $35(9)$ & Caramel Apple & $4 b, 4 b V$ \\
\hline $2010-2015$ & USA & $10(30)$ & Ice Cream & $1 / 2 b$ \\
\hline 2015 & USA & $30(10)$ & Soft Cheese & Unknown \\
\hline 2016 & USA & $19(5)$ & Packaged Greens & $4 b-V$ \\
\hline 2016 & USA & $9(33)$ & Frozen Vegetables & $1 / 2 \mathrm{a}$ \\
\hline
\end{tabular}

* https://www.cdc.gov/listeria/outbreaks/index.html

underlying health issues, and, except for one, all patients had isolates which were very closely related by Whole Genome Sequencing (WGS), though there were three different Pulsed Field Gel Electrophoresis (PFGE) patterns associated with these four cases. These four clinical isolates were found to be closely related by PFGE and WGS with the isolates obtained from unopened ice cream products made by a company in their Texas facility. Subsequently, a sampling of ice cream produced at other facilities within the company found $\mathrm{Lm}$ in another facility that was found to match 5 more cases in a retroactive analysis of listeriosis cases by PFGE and WGS dating back to January 2010. Investigation of the facility in Texas resulted in collection of large number of samples produced at that facility for further analysis to better understand the extent of contamination, the period of contamination, and the number of viable $\mathrm{Lm}$ present in these contaminated products. As ice cream is a no-growth food and Listeria survives well in ice cream under standard storage conditions ${ }^{16)}$, it was assumed that the enumeration of the products involved in the
Kansas City cases would provide fairly accurate information as to the number of organisms ingested by these patients. Table 2 summarizes the results of enumeration from three different ice cream products of which product $\mathrm{C}$ was the only one implicated in the Kansas City outbreak; however, all three products were produced on the same production line in the same facility. The data showed that all three products have a very low level of consistent contamination, the highest being in product $\mathrm{C}(99 \%)$ followed by product A $(77 \%)$ and product $\mathrm{B}(46 \%)^{17,18)}$. The enumeration was performed using a most probable number (MPN) method with a lower limit of detection of $0.03 \mathrm{MPN} / \mathrm{g}$, as well as a subset being evaluated by direct colony count. The data obtained with the product $\mathrm{C}$ was utilized to assess the dose response relationship as the $L m$ numbers in the enumerated samples were assumed to be representative of the numbers present at the time of exposure, especially given the consistency of the level of contamination observed ${ }^{17,19)}$. The analysis revealed that although the product was distributed widely 
Table 2. Ice cream samples, produced in one production line, enumerated to determine Listeria monocytogenes levels of contamination.

\begin{tabular}{cccccc}
\hline Product Type & $\begin{array}{c}\text { Samples } \\
\text { analyzed }\end{array}$ & Production dates & Percent contamination & $\begin{array}{c}\text { Median contamination } \\
\text { levels (MPN/g) }\end{array}$ & Reference \\
\hline A & 344 & May 2014-January 2015 & 77 & $0.07-3.8$ & Burall et al. ${ }^{18)}$ \\
B & 95 & December 2014-January 2015 & 46 & $0.02-0.64$ & Burall et al. ${ }^{18)}$ \\
C* & 2320 & November 2014-March 2015 & 99 & $0.15-7.1$ & Chen et al. ${ }^{17)}$ \\
\hline
\end{tabular}

*Product was linked to the cases in Kansas City, USA

and consumed by a broad cross section of the population in the USA, the absence of other reported listeriosis cases confirmed the previous assessments that the probability of developing listeriosis by the vast majority of the population from ingestion of low numbers of $L m$ is very small. However, the study indicated that the infectious dose, the minimum number of viable $L m$ to cause disease, could be very low for certain immunocompromised people, requiring additional risk assessment considerations to avoid such incidents.

\section{Recent Listeriosis Outbreaks Related to Produce}

Although the first major listeriosis outbreak in North America, was associated with cabbage ${ }^{20)}$ in 1980, in the following years, dairy and meat products became the main food vehicles responsible for most listeriosis outbreaks reported in the USA and elsewhere. Between 1998 and 2008, 24 listeriosis outbreaks were reported, and ready-to-eat meat products caused most of the outbreaks ${ }^{15)}$. Serotype $4 \mathrm{~b}$ was the single most prevalent serotype. However, among more recent outbreaks (Table 3), ready-to-eat meats have played a less prominent role as vehicles of infection, while dairy products have remained a prominent cause; importantly, produce-related outbreaks have been reported with increasing frequency in recent years. These outbreaks also indicate that other serotypes, e.g. 1/2a, 1/2b and a few recent cases of $4 \mathrm{bV}$, also contribute to these outbreaks. It is interesting to note that with the implementation of PFGE- and WGSbased typing systems, we are discovering more outbreaks and discovering them earlier, resulting in fewer cases than the outbreaks before the PulseNet era. Recently, Burall et al. ${ }^{21)}$ described a series of outbreaks involving caramel apple, cheese, stone fruit and bagged salad and showed that all these outbreaks/incidents were caused by serotype $4 \mathrm{bV}$, though the caramel apple outbreak also involved cases caused by serotype $4 \mathrm{~b}$ strains. The most interesting is the caramel apple outbreak $^{22)}$, which caused 35 illnesses and seven deaths encompassing 12 states (Table 3). The outbreak was caused by two different related serotypes, $4 \mathrm{~b}$ and $4 \mathrm{bV}$, a feature also observed during 2011 cantaloupe outbreak where 1/2a and $1 / 2 b$ were involved ${ }^{23)}$. The caramel apple outbreak also included three cases, two caused by $4 \mathrm{bV}$ strains, between 3-15 years of age without any recognized causes of underlying immune compromise and one male between 18-25 years of age with no disclosed pre-disposing conditions. This was very unusual given the demographics of listeriosis cases are comprised mostly of elderly and immunocompromised patients ${ }^{15)}$. A series of WGS analysis of the $4 \mathrm{bV}$ isolates from these outbreaks by JSpecies, Center for Food Safety and Nutrition (CFSAN) WGS Pipeline and Basic Local Alignment Search Tool (BLAST) analysis also revealed that these isolates belonged to a distinct clade and were genetically well separated from $4 \mathrm{~b}$ and $4 \mathrm{bV}$ strains reported from other outbreaks, including $4 \mathrm{~b}$ isolates recovered from the caramel apple-associated outbreak ${ }^{21)}$. All three different analytical tools resulted in a very similar conclusion although the Single Nucleotide Polymorphism (SNP) analyses provided much more resolution in the analysis. Another significant finding from that work ${ }^{21)}$ was the observation with Multi Virulence Loci Sequence Typing (MVLST) analysis, utilizing a limited set of defined virulence gene sequences, that selective pressures on a subset of genes may have played a significant role in our ability to understand the strain-to-strain variability and thus the evolution of newer traits. Furthermore, the analysis reported by Burall et al. ${ }^{21)}$ clearly showed that these highly related $4 \mathrm{bV}$ strains may have originated from a small geographical region in the USA. Whether this was due to shared agricultural practices, supplies, and/or equipment leading to cross-contamination or due to the natural presence of this strain throughout this geographical region is not clear at this point. It is, however, very clear that these $4 \mathrm{bV}$ strains, because of their involvement in these recent outbreaks, may represent an emergent clade and must be monitored carefully and studied in-depth to understand their role in listeriosis disease burden.

\section{Conclusions}

Although improved food production practices and educa- 
Table 3. Multistate Outbreaks in the USA since 2011.

\begin{tabular}{lccccc}
\hline Year & Food & Cases & States & Hospitalization & Death \\
\hline 2011 & Cantaloupe & 147 & 28 & 143 & 33 \\
2012 & Cheese & 22 & 14 & 20 & 4 \\
2013 & Cheese & 6 & 5 & 6 & 1 \\
2014 & Cheese & 8 & 2 & 7 & 1 \\
2014 & Cheese & 5 & 4 & 4 & 2 \\
2014 & Bean sprouts & 5 & 2 & 5 & 7 \\
2014 & Caramel apple & 35 & 12 & 34 & 3 \\
2015 & Ice cream* & 10 & 4 & 10 & 3 \\
2015 & Soft cheese & 30 & 10 & 28 & 1 \\
2016 & Packaged salad & 19 & 9 & 19 & 1 \\
2016 & Raw milk & 2 & 2 & 2 & 3 \\
2016 & Frozen vegetables & 9 & 4 & 9 & 2 \\
2017 & Soft raw milk cheese & 8 & 4 & 8 & 2 \\
\hline
\end{tabular}

* Number includes cases from a retrospective study

tion campaigns are likely to have reduced the incidence of listeriosis in the USA in recent decades, recent outbreaks have provided important insights into this pathogen. Increasing outbreaks/cases related to fresh produce and continuing dairy-related cases indicate much work remains to bring about further reductions in incidence. The 2015 ice cream outbreak showed that a non-growth ready-to-eat food with very low numbers of $\mathrm{Lm}$ can pose a substantial threat to subsets of immunocompromised persons, reinforcing the concept that the infectious dose for $\mathrm{Lm}$ should be considered as a function of the population exposed rather than a single number for all ${ }^{24}$. This emphasizes the value of enumerating outbreak-related samples as they provide valuable information regarding infectious doses in different exposed populations. Better in vivo models, e.g. Galleria mellonella ${ }^{25)}$, together with improved understanding of role of food matrices in the infection process may further help to prevent human listeriosis. The ice cream outbreak also highlighted that $\mathrm{Lm}$ in the ready-to-eat food processing environment must be strictly monitored, and all efforts should be taken to eliminate the burden of $\mathrm{Lm}$ in production environments.

The recent outbreaks involving a clade of $4 \mathrm{bV}$ strains indicate that this genotype may be an emerging clade of $\mathrm{Lm}$. Although we know this clade is genetically different from the other $4 \mathrm{~b}$ and $4 \mathrm{bV}$ strains associated with different listeriosis outbreak, their biological and physiological significance in terms of disease-causing potential and environmental sur- vival are not clear. Further research is needed to understand these properties. It is, however, clear that continuous monitoring of outbreak isolates and analysis by WGS is needed to identify the emergence of new clones or clades of $\mathrm{Lm}$ that may present increased risks to public health.

\section{Conflict of interest}

The authors have no conflict of interest.

\section{References}

1. Ooi ST, Lorber B. Gastroenteritis due to Listeria monocytogenes. Clin Infect Dis. 2005; 40: 1327-1332. doi: 10.1086/429324. PMID: 15825036

2. Scallan E, Hoekstra RM, Angulo FJ, et al. Foodborne illness acquired in the United States--major pathogens. Emerg Infect Dis. 2011; 17: 7-15. doi: 10.3201/eid1701.P11101. PMID: 21192848

3. HHS Reduce infections caused by Listeria monocytogenes transmitted commonly through food. In: Healthy People 2020 FS, editor. 2014. p. FS-13.

4. Lado BH, Yousef AE. Characteristics of Listeria monocytogenes important to food processors. In: Ryser ET, Marth EH, editors. Listeria, listeriosis and food safety. Third ed. Boca Raton (FL): CRC Press; 2007. p. 157-214.

5. Orsi RH, Wiedmann M. Characteristics and distribution of Listeria spp., including Listeria species newly described since 2009. Appl Microbiol Biotechnol. 2016; 100: 5273-5287. doi: 10.1007/s00253-016-7552-2. PMID: 27129530 
6. Seeliger HP, Hohne K. Serotyping of Listeria monocytogenes and Related Species. In: Bergan TN, J.R., editor. Methods in Microbiology. 131979. p. 31-49.

7. Datta AR. Listeria monocytogenes. In: Miliotis MD, Bier JW, editors. International Handbook of Foodborne Pathogens. New York: Marcel Dekker, Inc.; 2003. p. 105-21.

8. Doumith M, Buchrieser C, Glaser P, Jacquet C, Martin P. Differentiation of the major Listeria monocytogenes serovars by multiplex PCR. J Clin Microbiol. 2004; 42: 3819-3822. doi: 10.1128/JCM.42.8.3819-3822.2004. PMID: 15297538

9. Burall LS, Simpson AC, Datta AR. Evaluation of a serotyping scheme using a combination of an antibody-based serogrouping method and a multiplex PCR assay for identifying the major serotypes of Listeria monocytogenes. J Food Prot. 2011; 74: 403-409. doi: 10.4315/0362-028X.JFP-10-355. PMID: 21375876

10. Laksanalamai P, Huang B, Sabo J, et al. Genomic characterization of novel Listeria monocytogenes serotype $4 \mathrm{~b}$ variant strains. PLoS One. 2014; 9: e89024. PMID: 24586485

11. Leclercq A, Chenal-Francisque V, Dieye H, et al. Characterization of the novel Listeria monocytogenes PCR serogrouping profile IVb-v1. Int J Food Microbiol. 2011; 147: 74-77. doi: 10.1016/j.ijfoodmicro.2011.03.010. PMID: 21470706

12. Laksanalamai P, Jackson SA, Mammel MK, Datta AR. High density microarray analysis reveals new insights into genetic footprints of Listeria monocytogenes strains involved in listeriosis outbreaks. PLoS One. 2012; 7: e32896. doi: 10.1371/ journal.pone.0032896. PMID: 22457724

13. D'Orazio SE. Animal models for oral transmission of Listeria monocytogenes. Front Cell Infect Microbiol. 2014; 4: 15. PMID: 24575393

14. Chen Y, Gonzalez-Escalona N, Hammack TS, Allard MW, Strain EA, Brown EW. Core genome multilocus sequence typing for the identification of globally distributed clonal groups and differentiation of outbreak strains of Listeria monocytogenes. Appl Environ Microbiol. 2016; 82: 6258-6272. doi: 10.1128/AEM.01532-16. PMID: 27520821

15. Cartwright EJ, Jackson KA, Johnson SD, Graves LM, Silk BJ, Mahon BE. Listeriosis outbreaks and associated food vehicles, United States, 1998-2008. Emerg Infect Dis. 2013; 19: 1-9, quiz 184. doi: 10.3201/eid1901.120393. PMID: 23260661
16. Gougouli M, Angelidis AS, Koutsoumanis K. A study on the kinetic behavior of Listeria monocytogenes in ice cream stored under static and dynamic chilling and freezing conditions. J Dairy Sci. 2008; 91: 523-530. doi: 10.3168/jds.20070255. PMID: 18218738

17. Chen YI, Burall LS, Macarisin D, et al. Prevalence and Level of Listeria monocytogenes in Ice Cream Linked to a Listeriosis Outbreak in the United States. J Food Prot. 2016; 79: 1828 1832. doi: 10.4315/0362-028X.JFP-16-208. PMID: 28221903

18. Burall LS, Chen Y, Macarisin D, et al. Enumeration and characterization of Listeria monocytogenes in novelty ice cream samples manufactured on a specific production line linked to a listeriosis outbreak. Food Contr. 2017; 82: 1-7. doi: 10.1016/j.foodcont.2017.06.026.

19. Pouillot R, Klontz KC, Chen Y, et al. Infectious Dose of Listeria monocytogenes in Outbreak Linked to Ice Cream, United States, 2015. Emerg Infect Dis. 2016; 22: 2113-2119. doi: 10.3201/eid2212.160165. PMID: 27869595

20. Evans JR, Allen AC, Stinson DA, Bortolussi R, Peddle LJ. Perinatal listeriosis: report of an outbreak. Pediatr Infect Dis J. 1985; 4: 237-241. doi: 10.1097/00006454-19850500000005. PMID: 4000986

21. Burall LS, Grim CJ, Datta AR. A clade of Listeria monocytogenes serotype $4 \mathrm{~b}$ variant strains linked to recent listeriosis outbreaks associated with produce from a defined geographic region in the US. PLoS One. 2017; 12: e0176912. doi: 10.1371/ journal.pone.0176912. PMID: 28464038

22. Angelo KM, Conrad AR, Saupe A, et al. Multistate outbreak of Listeria monocytogenes infections linked to whole apples used in commercially produced, prepackaged caramel apples: United States, 2014-2015. Epidemiol Infect. 2017; 145: 848856. doi: 10.1017/S0950268816003083. PMID: 28065170

23. Laksanalamai P, Joseph LA, Silk BJ, et al. Genomic characterization of Listeria monocytogenes strains involved in a multistate listeriosis outbreak associated with cantaloupe in US. PLoS One. 2012; 7: e42448. doi: 10.1371/journal. pone.0042448. PMID: 22860127

24. Pouillot R, Hoelzer K, Chen Y, Dennis SB. Listeria monocytogenes dose response revisited--incorporating adjustments for variability in strain virulence and host susceptibility. Risk Anal. 2015; 35: 90-108. PMID: 24975545

25. Mukherjee K, Altincicek B, Hain T, Domann E, Vilcinskas A, Chakraborty T. Galleria mellonella as a model system for studying Listeria pathogenesis. Appl Environ Microbiol. 2010; 76: 310-317. doi: 10.1128/AEM.01301-09. PMID: 19897755 